\title{
Cellular Therapy, a Novel Treatment Option for Intellectual Disability: A Case Report
}

\author{
Sharma A ${ }^{1}$, Sane H $^{2}$, Pooja Kulkarni ${ }^{2 *}$, Akshya Nagrulkar ${ }^{3}$, Nandini Gokulchandran ${ }^{1}$, Prerna Badhe, and Akshata Shetty ${ }^{3}$ \\ ${ }^{1}$ Department of Medical Services and Clinical research, NeuroGen Brain and Spine Institute, India \\ ${ }^{2}$ Department of Research and Development, NeuroGen Brain and Spine Institute, India \\ ${ }^{3}$ Department of NeuroRehabilitation, NeuroGen Brain and Spine Institute, India
}

\begin{abstract}
Intellectual Disability is a non progressive developmental condition which significantly affects cognition, learning and adaptive behavior of an individual. Currently, there is no treatment available for intellectual disability. However, the therapies available focus mainly on the symptoms of the patient and do not address its core neuropathology. Neurorestorative strategies such as cellular therapy may benefit in these disorders. To study the effect of cellular therapy in intellectual disability, we administered a 13 year old boy with autologous bone marrow mononuclear cells, intrathecally. As a part of the protocol, he was also put on a personalized rehabilitation program. Follow up was done at 3 months and 6 months. No major adverse events were recorded post intervention. In a period of 6 months, he showed improved eye contact, cognition, learning ability, behavior and ability to perform activities of daily living. His score on Functional Independence Measure increased from 67 to 76 . On comparing the pre and post PET CT scan, improvement in metabolic activity of hippocampus, left amygdala and cerebellum was recorded. These changes correlated to the functional outcome. These changes suggest that cellular therapy in combination with rehabilitation may repair the neuronal networks in intellectual disability, hence improving processing of information. It was found to be a safe and effective therapeutic modality. Hence, cellular therapy may provide hope for patients and caregivers to improve their quality of life.
\end{abstract}

Keywords: Intellectual disability; Cellular therapy; Autologous; Bone marrow mononuclear cells; PET CT scan

\section{Introduction}

Intellectual Disability (ID) is defined as "a group of developmental conditions which include significant cognitive impairment along with learning disability and difficulty in adaptive behavior and skills." [1]. It can result due to a variety of causes such as genetic (Fragile $\mathrm{X}$ chromosome syndrome, Down syndrome, Lesch-Nyhan syndrome, adrenoleukodystrophy, etc) congenital (neonatal hypothyroidism, heavy metal poisoning, infections), developmental (during prenatal, perinatal or post natal period) and environmental factors [2]. These factors may result in functional abnormalities of the Central Nervous System (CNS) with varying severity. Based on the severity it is categorized as Mild, Moderate, Severe and Profound. (DSM-V) It is primarily diagnosed through a comprehensive patient and family history and intelligence is assessed on the basis of an IQ test. According to the World Health Organization (WHO), the IQ of an individual with intellectual disability is equal to or less than 70 [3].

Due to inability of the neurons to instinctively repair themselves [4], there is no cure for mental retardation as yet. The conventional treatment strategies such as occupational therapy, speech therapy, behavior therapy currently available for ID are aimed towards specific symptoms [5]. Their objective is to maintain and reduce the level of impairment and enhance the functional status of the affected individual. However, these strategies do not address the underlying neuropathology. To reverse the damage caused to the CNS, a neurorestorative therapy such as cellular therapy could be beneficial. We report a case of ID who was administered with autologous bone marrow mononuclear cells (BMMNCs) in combination with rehabilitation.

\section{Case Report}

A 13 year old male child, born full term normal delivery and cried immediately after birth, had a history of neonatal jaundice. His developmental milestones were delayed with speech at 5 years. He also had febrile seizures at 1 year of age after which he was diagnosed to have intellectual disability with seizure disorder.
On examination, he was hypotonic and hyporeflexic. He had good voluntary control for his upper and lower limbs but had difficultyin sitting and standing erect. Balance and hand functions were fair. On sensory evaluation, he was tactile hypersensitive, vestibular hypersensitive and propioceptive hyposensitive. He was hyperactive, aggressive, had inconsistent eye contact, repetitive behavior, mood swings, poor cognition and visual perceptual skills, difficulty in adjusting to a new place and made unusual noises. Attention and concentration was mildly affected. He required maximum assistance in eating, grooming, bathing and dressing while minimal assistance in toileting. He had modified independence in bladder management, transfer, locomotion. He needed moderate assistance in communication, social interaction. However, had maximal dependence in problem solving and memory? He scored 67 on Functional Independence Measure (FIM). His IQ was calculated to be 47 suggesting moderate intellectual disability.

MRI Brain showed bilateral Pachygyria. PET-CT scan showed reduced FDG uptake in the mesial temporal structures, amygdala, hippocampus and left cerebellum. Pachygyria was identified bilaterally with altered FDG uptake.

\section{Material and Methods}

Patient selection was based on the World Medical Associations Helsinki declaration [6]. The protocol was reviewed and approved

\begin{abstract}
*Corresponding author: Pooja Kulkarni, Department of Research and Development, NeuroGen Brain and Spine Institute- StemAsia Hospital and Research Centre, Navi Mumbai 400706, India, Tel: +9122-25283706/ +9122-41136565; Email: poojakul28@gmail.com
\end{abstract}

Received October 20, 2014; Accepted January 26, 2015; Published January 28 2015

Citation: Sharma A, Sane H, Pooja K, Akshya N, Nandini G, et al. (2015) Cellular Therapy, a Novel Treatment Option for Intellectual Disability: A Case Report. J Clin Case Rep 5: 483. doi:10.4172/2165-7920.1000483

Copyright: @ 2015 Sharma A, et al. This is an open-access article distributed under the terms of the Creative Commons Attribution License, which permits unrestricted use, distribution, and reproduction in any medium, provided the original author and source are credited. 
by Institutional Committee for Stem Cell Research and Therapy (ICSCRT). An informed consent was obtained from the parents. Patient underwent serological, biochemical and hematological blood tests, PET-CT scan of brain, Magnetic Resonance Imaging (MRI) of brain, electroencephalography (EEG), and electrocardiography a week before cellular therapy.

Granulocyte Colony Stimulating Factor (GCSF) was administered $48 \mathrm{hrs}$ and $24 \mathrm{hrs}$ before the transplantation to maximize the quantity of mononuclear cells in bone marrow. On the day of transplantation as the patient lay in supine position, sedation with local anesthesia was administered in the region of the right anterior superior iliac spine. $100 \mathrm{ml}$ of bone marrow was aspirated using the bone marrow aspiration needle and collected in heparinized tubes. The aspirate was then transferred to the laboratory. In the stem cell laboratory the MNCs were separated by the density gradient method. The cells were checked for CD34+ counts by Fluorescence Activated Cell Sorter (FACS) analysis and the viability was $98 \%$ in this patient. The cells were injected intrathecally at the level of L4-L5. $3 \times 10^{8}$ cells were injected. Simultaneously, Methyl Prednisolone $1 \mathrm{gm}$ in $500 \mathrm{~mL}$ Ringer Lactate was given intravenously to prevent local inflammation.

As a part of the protocol, followed by cellular therapy the patient was given intensive neurorehabilitation therapy. It included a multidisciplinary rehabilitation protocol comprising of occupational therapy, speech therapy, psychotherapy, and diet advice. The Occupational therapy interventions included both restorative and adaptive strategies to improve performance of the Activities of Daily Living (ADL) tasks the participants defined as relevant and meaningful. Speech therapists helped with pronunciation of words, making sounds, pitch control and improve communication skills. Psychological counseling was conducted for the patient as well as family members. A personalized home program was given to the patient and was advised to continue rehabilitation for 6 months. He was followed up at 3 months and 6 months post intervention. At 6 months post intervention a detailed reassessment was carried out and repeat PET-CT scan was obtained.

\section{Results}

At 3 months follow up, his body awareness had improved and he could recognize all body parts. His attention span had increased; eye contact improved and could catch the ball while playing. His imitation skills, communication and expression had improved; he could identify and name pictures. He was able to place $\mathrm{A}$ to $\mathrm{Z}$ pegs in its place. He had learnt names of vegetables and fruits. His aggressive behavior and temper tantrums had reduced. The ability to perform daily activities had improved. He could brush his teeth, comb hair, wear his shirt by himself, and also eat independently. At 6 months follow up, his spatial relation had improved along with speech. His learning abilities improved as he could now recognize English alphabets and also understand the concept of directions. He could identify different vegetables and fruits, his cognitive skills were better than before. He started seeking attention. He initiated to bathe independently. He could hold the pencil and write numbers. His FIM was improved from 67 to 76 .

On repeating the PET CT scan and comparing after 6 months of intervention there was improvement in metabolic activity involving hippocampus, left amygdala and left cerebellum (Figure 1).
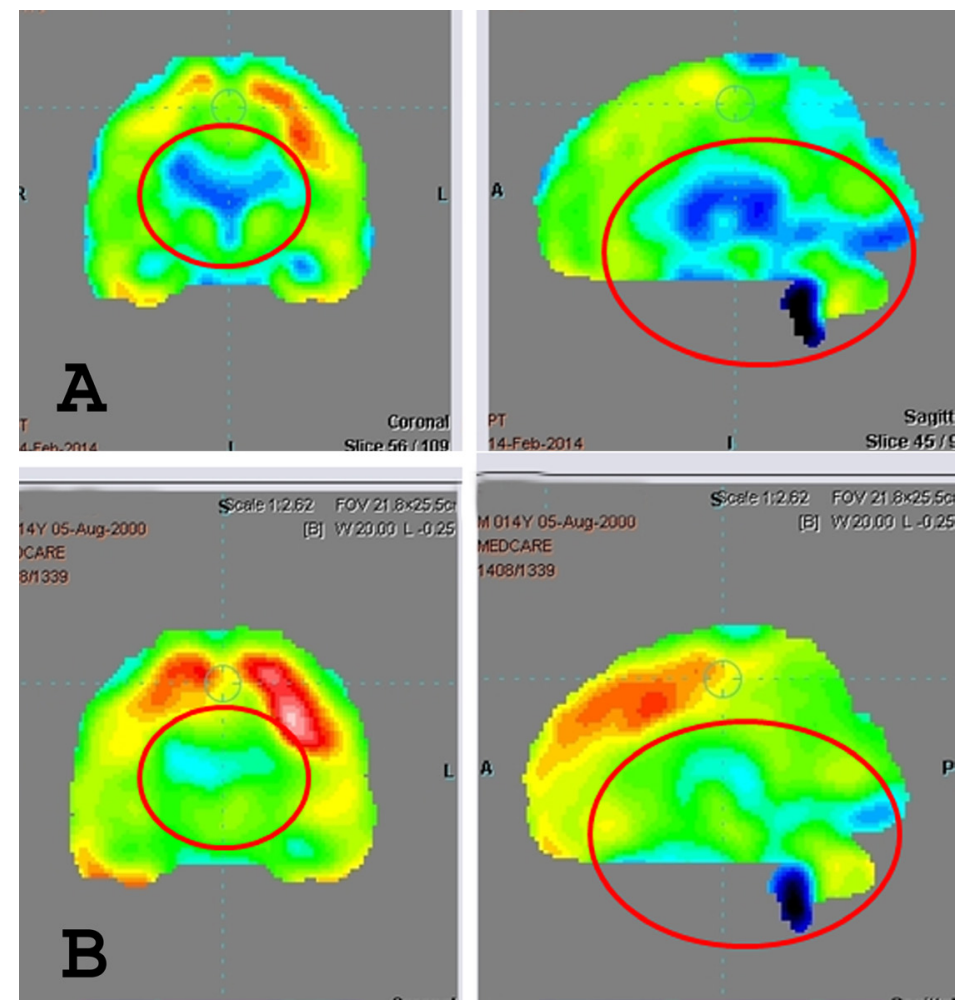

Figure 1: (A)PET-CT scan images pre stem cell therapy (A) and post stem cell therapy. (B) (Colour code: black- severe hypometabolism, blue- hypometabolism, greennormal metabolism): The encircled areas depict the hypometabolic areas (blue and black areas) which have improved post intervention. Improvement in metabolic activity of hippocampus, left amygdala and left cerebellum is seen. 


\section{Discussion}

In the past decade, cellular therapy as a treatment strategy has shown promising results in various incurable neurodevelopmental disorders. With a growing prevalence rate of intellectual disability it is imperative to explore new avenues of management. To study the effect of cellular therapy in ID, we administered a case of ID with autologous bone marrow mononuclear cells, intrathecally. Bone marrow is a rich source of cells. These cells are easily to obtain and have no ethical or moral issues associated with its use. Mononuclear cells obtained from the bone marrow are a mixture of various progenitor cells. Studies have shown that using a mixture of cells is more beneficial than using individual fractions of cells [7]. Since, these cells are autologous in nature they eliminate any possibility of immune rejection. Intrathecal route of administration is one of the minimally invasive interventions. The cells are injected into the CSF which possesses properties supporting cell survival and growth [8].

Evidence suggests that the underlying neuropathologies in intellectual disability are neuronal death and alterations in neuronal networks, brain plasticity, cell migration, cell multiplication, axon growth, synaptogenesis, dendritic arborization, etc. [9] Studies have also demonstrated abnormalities majorly in hippocampus and cerebral cortex amongst other areas of the brain. These changes in the brain lead to defect in information processing further leading to affected cognition and adaptive behavior [10]

BMMNCs have a unique property of migrating towards the damaged areas on administration. Experiments have shown that these cells survive, migrate, proliferate and differentiate into the required cell types [11]. Cellular transplantation not only helps in replacement of the lost cells but also support the endogenous cells. They stimulate the existing cells and prevent further damage. Simultaneously, they also carry out the repair process through paracrine mechanisms including secretion of growth factors, angiogenesis, neurogenesis, immunomodulation, decreasing inflammation, etc. [12]. Experimental studies have shown that bone marrow derived cells are capable of differentiating into mature neurons or glial cells $[13,14]$. In animal models, these cells have improved the neurological deficit by generating neural cells $[15,16]$. These findings increase the likelihood of BMMNCs being therapeutically beneficial for patients with neurological conditions. Before cellular transplantation, the patient was administered with G-CSF as it stimulates the proliferation of stem cells from the bone marrow. It also exhibits neuroprotective activity by inhibiting neuronal apoptosis and inflammation [17].

Along with cellular therapy, the patient was given neurorehabilitation as a part of the protocol. Evidence suggests that exercise augments the effect of cellular therapy. Exercise stimulates the injected cells and directs them to perform particular functions $[18,19]$.

In this case, 6 months after the intervention, the patient showed substantial improvement in various functions such as cognition, learning ability, activities of daily living, etc. Along with these functional improvements there was a positive change in the PET CT scan. A PET CT scan measures the FDG uptake of various brain areas which correlates with the level of functionality of neurons. Thus, improved metabolism indicates increased function of the previously damaged cells. On repeating the PET CT scan after the intervention and comparing it to the previous scan, increase in metabolic activity of hippocampus, left amygdala and left cerebellum areas of brain was observed. These areas showed reduced metabolism before the intervention. PET CT scan identifies changes at a cellular level and hence, it can be used as an effective monitoring tool for cellular transplantation. The changes on the PET CT scan correlated to the functional outcome.

Increased metabolic activity in hippocampus can be correlated clinically with improvement in learning ability. The patient was able to learn new things, such as learn new activities of daily living [20]. Increased metabolic activity in amygdala was responsible for improvement in cognitive skills, emotional learning and decision making [21]. Improvement in balance, coordination and fine motor activities can be correlated to the increased metabolism in cerebellum [22]. His FIM score improved from 67 to 76 in a period of 6 months improving functional independence.

\section{Limitations}

The reported case is a single case report hence larger studies are required to prove the efficacy of cellular therapy in ID. One of the limitations of this study is that the genetic work up for this case is not available to confirm the presence of a genetic defect. Also, there was no control available to study the independent effect of rehabilitation or cellular therapy.

\section{Conclusion}

All the clinical and objective changes in the patient suggest that cellular therapy along with rehabilitation could repair the underlying neuropathologies in ID, hence improving processing of information. Cellular transplantation was found to be safe, non toxic and effective in this case with no major adverse events. Although ID has been considered as a permanent brain disorder, cellular therapy may provide a hope for patients and caregivers to improve their quality of life. However, more studies are required to demonstrate long term benefits of cellular transplantation in ID.

\section{References}

1. Salvador-Carulla L, Reed GM, Vaez-Azizi LM, Cooper SA, Martinez-Leal R, et al. (2011) Intellectual developmental disorders: towards a new name, definition and framework for "mental retardation/intellectual disability" in ICD-11. World Psychiatry 10:175-180.

2. Katz G, Lazcano Ponce E (2008) Intellectual disability: definition, etiological factors, classification, diagnosis, treatment and prognosis. Salud pública Mex s132-s141.

3. Liana K, Muhammad A, John BV (2000) The genetic basis of non-syndromic intellectual disability: a review American Psychiatric Association. J Neurodev Disord. 2: 182-209.

4. Gogel S, Gubernatorand M, Minger SL (2011) Progress and prospects: stem cells and neurological disease. Gene Therapy 18: 1-6

5. Kottorp A, Hallgren M, Bernspang B, Fisher AG (2003) Client Centred Occupational Therapy for Persons with Mental Retardation: Implementation of an Intervention Programme in Activities of Daily Living Tasks. Scandanavian Journal of Occupational Therapy 10: 51-60.

6. Carlson RV, Boyd KM, Webb DJ. (2004) The revision of the declaration of Helsinki: past, present and future. British Journal of Clinical Pharmacology 57: 695-713.

7. Yoon SH, Shim YS, Park YH, Chung JK, Nam JH, et al. (2007). Complete Spinal Cord Injury Treatment Using Autologous Bone Marrow Cell Transplantation and Bone Marrow Stimulation with Granulocyte Macrophage-Colony Stimulating Factor: Phase I/II Clinical Trial. Stem Cells. 25: 2066-2073.

8. Miyan JA, Zendah M, Mashayekhi F, Owen-Lynch PJ (2006) Cerebrospina fluid supports viability and proliferation of cortical cells in vitro, mirroring in vivo development. Cerebrospinal Fluid Research. 3.

9. Dierssen M, Ramakers GJA (2006) Dendritic pathology in mental retardation: from molecular genetics to neurobiology. Genes, Brain and Behavior 5: 48-60.

10. Chelly J, Khelfaoui M, Francis F, Cherif B, Bienvenu T (2006) Genetics and pathophysiology of mental retardation. European Journal of Human Genetics 14: 701-713. 
Citation: Sharma A, Sane H, Pooja K, Akshya N, Nandini G, et al. (2015) Cellular Therapy, a Novel Treatment Option for Intellectual Disability: A Case Report. J Clin Case Rep 5: 483. doi:10.4172/2165-7920.1000483

11. Chen RJ, Cheng GY, Sheu CC, Tseng GF, Wang TJ, et al. (2008) Transplanted bone marrow stromal cells migrate, differentiate and improve motor function in rats with experimentally induced cerebral stroke. J Anat 213: 249-258.

12. Gnecchi M, Zhang Z, Ni A, Dzau AJ (2008) Paracrine mechanisms in adult stem cell signaling and therapy. Circulation Research 103:1204-1219

13. Munoz-Elias G, Woodbury D, Black IB (2003) Marrow stromal cells, mitosis and neuronal differentiation: Stem cell and precursor functions. Stem Cells 21: 437-448

14. Sanchez-Ramos J, Song S, Cardozo-Pelaez F, Hazzi C, Stedeford, T, et al. (2000) Adult bone marrow stromal cells differentiate into neural cells in vitro. Exp. Neurol 164: 247- 256.

15. Chopp M, Zhang XH, Li Y, Wang L, Chen J, et al. (2000) Spinal cord injury in rat: Treatment with bone marrow stromal cell transplantation. Neuroreport 11: 3001-3005

16. Akiyama Y, Radtke C, Kocsis JD (2002) Remyelination of the rat spinal cord by transplantation of identified bone marrow stromal cells. J. Neurosci 22: 66236630

17. Doycheva D, Shih G, Chen H, Applegate R, Zhang JH, et al. (2013) Granulocyte- colony stimulating factor in combination with stem cell factor confers greater neuroprotection after hypoxic-ischemic brain damage in the neonatal rats than a solitary treatment. Translational stroke research 4: 171-178.

18. Van Haren IE, Timmerman H, Potting CM, Blijlevens NM, Staal JB, et al. (2013) Physical exercise for patients undergoing hematopoietic stem cell transplantation: systematic review and meta-analyses of randomized controlled trials. Phys Ther. 93: 514-528.

19. Teng YD, Liao WL, Choi H, Konya D, Sabharwal S, et al. (2006) Physical activity mediated functional recovery after spinal cord injury: potential roles of neural stem cells. Regenerative Medicine 1: 763-776.

20. Whishaw IQ, Jarrard LE (1996) Evidence for extrahippocampal involvement in place learning and hippocampal involvement in path integration. Hippocampus 6: $513-524$

21. Sartor M, Markowitsch HJ (1985) Involvement of the amygdala in learning and memory: A critical review, with emphasis on anatomical relations. Behavioral Neuroscience. 99: 342-380.

22. Gillig PM, Sanders RD (2010) Psychiatry, Neurology, and The Role Of The Cerebellum. Psychiatry 7: 38-43. 